ORIGINAL ARTICLE

\title{
Cancers and immune related diseases associated with Down's syndrome: a record linkage study
}

\author{
M J Goldacre, C J Wotton, V Seagroatt, D Yeates
}

Arch Dis Child 2004;89:1014-1017. doi: 10.1136/adc.2003.046219

See end of article for authors' affiliations

.....................

Correspondence to:

Professor Michael

$\mathrm{J}$ Goldacre, Unit of

Health-Care

Epidemiology, Department

of Public Health, University

of Oxford, Old Road,

Oxford OX3 7LF, UK'

michael.goldacre@dphpc.

ox.ac.uk

Revised version received 3 March 2004

Accepted for publication

5 April 2004
Objective: To determine the risk of cancers and selected immune related diseases in people with Down's syndrome, relative to risk in other people.

Design: Cohort analysis of a linked dataset of abstracts of hospital and death records; results expressed as the ratios of rates of disease in people with and without Down's syndrome.

Setting: The former Oxford health region, England, 1963-1999.

Subjects: Cohort of 1453 people with Down's syndrome and cohort of 460000 people with other conditions for comparison.

Main outcomes: As expected, the rate ratio for leukaemia was substantially elevated in people with Down's syndrome: it was 19-fold higher (95\% confidence intervals 10.4 to 31.5 ) than the rate in the comparison cohort. For other cancers combined, excluding leukaemia, the rate ratio was not significantly elevated (1.2; 0.6 to 2.2). The risk of testicular cancer was increased (12.0; 2.5 to 35.6), although this was based on only three cases in the cohort of subjects with Down's syndrome. Significantly elevated risks were found for coeliac disease $(4.7 ; 1.3$ to 12.2$)$, acquired hypothyroidism $(9.4 ; 3.4,20.5)$, other thyroid disorders, and type 1 diabetes mellitus $(2.8 ; 1.0$ to 6.1). A decreased risk was found for asthma $(0.4 ; 0.2$ to 0.6$)$.

Conclusions: Our data add to the body of information on the risks of co-morbidity in people with Down's syndrome. The finding on asthma needs to be confirmed or refuted by other studies. l: is well established that the risk of leukaemia in people with Down's syndrome is much higher than that in the general population. ${ }^{12}$ It is less clear whether people with Down's syndrome have an elevated risk of other cancers. While it is unlikely that the risk of other cancers is high-if it were, it would be clinically apparent - a modest elevation of risk remains possible. For example, a link with testicular cancer has been suggested. ${ }^{134}$ There is also some evidence that the prevalence of coeliac disease, thyroid disorders, and diabetes mellitus is higher in people with Down's syndrome than in the general population..$^{5-8}$ The authors of a recent review of non-malignant diseases associated with Down's syndrome suggested that coeliac disease and hypothyroidism occurred frequently enough to warrant screening (annually for hypothyroidism and a one-off screen at 24 months for coeliac disease). ${ }^{9}$ They also suggested that diabetes mellitus occurs more frequently among people with Down's syndrome than in the general population.

We used a database of linked statistical records, in a large defined population, to add to the evidence about whether cancers and a range of diseases with an autoimmune component are more common in people with Down's syndrome than in others.

\section{METHODS}

\section{Population and data}

We used data from the Oxford Record Linkage Study (ORLS). ${ }^{10}$ The ORLS includes brief statistical abstracts of records of all hospital admissions, including day cases, in National Health Service (NHS) hospitals, and all deaths regardless of where they occurred, in defined populations within the former Oxford NHS Region from January 1963 to March 1999. The hospital data were collected routinely in the NHS for all types of hospital as the region's hospital discharge statistics. The death data derive from death certificates. Data collection covered two health districts and their associated hospitals from 1963 (population 850 000), six districts from 1975 (population 1.9 million), and all districts and hospitals in the region from 1987 (population 2.5 million). The data for each individual were linked together routinely as part of the region's health information system. They are anonymised and archived.

The Down's syndrome cohort was obtained by identifying statistical records of individuals who had been admitted to NHS hospitals for the condition. A reference cohort was constructed by selecting records of admission for various other medical and surgical conditions. This is based on our reference group of conditions that has been used in other studies of inter-relationships between diseases. ${ }^{10}$ We searched the database for any subsequent NHS hospital care, or death, for cancers and immune related diseases in these two cohorts. We considered that rates of cancer and the immune related diseases in the reference cohort would approximate to those in the general population of the region while allowing for migration in and out of it (data on migration of individuals were not available). In our main analyses, we excluded people when the outcome disease (for example, cancer) occurred at the first recorded admission for Down's syndrome or reference condition. We did this to avoid the bias of identifying people for the Down's syndrome cohort because they had cancer. However, we also repeated the analyses including those whose outcome condition (for example, cancer) occurred at the first recorded admission for either Down's syndrome or reference condition; and we also comment briefly on these analyses when their inclusion made any appreciable difference to the rate ratios.

Abbreviations: $95 \% \mathrm{Cl}, 95 \%$ confidence intervals; NHS, National Health Service; ORLS, Oxford Record Linkage Study 


\section{Statistical methods}

We calculated rates of each cancer or immune related disease based on person-years at risk. We took date of entry into each cohort as date of first admission for Down's syndrome or reference condition, and date of exit for each individual disease as the date of subsequent admission for the disease (if any occurred), death, or 31 March 1999, whichever was the earliest. In comparing the Down's cohort with the reference cohort, we first calculated rates, standardised by age (in 5 year age groups for those aged 5 years and over, and in 1 year age groups for those aged less than 5 years), sex, calendar year of first recorded admission, and district of residence, taking the combined Down's and reference cohorts as the standard population. We then calculated the ratio of the standardised rate of occurrence in the Down's cohort relative to that in the reference cohort. The confidence interval for the rate ratio and $\chi^{2}$ statistics for its significance were calculated as described elsewhere. ${ }^{11}$

In comparing the Down's and reference cohorts, the precision of the rate ratio depends on the number of people with each subsequent disease within each cohort. The size of the Down's cohort is fixed by the number in the database with the condition. In the reference cohort, we included all the people in the database with the comparison conditions in each age group. We did this to maximise the numbers in each stratum in the reference cohort in order to maximise the precision of the rate ratios.

\section{RESULTS}

There were 1453 individuals in the Down's syndrome cohort and over 460000 in the comparison cohort. Table 1 shows the age distribution of Down's syndrome patients and the reference cohort, at the time of first recorded admission. The median age at first admission for Down's syndrome was 3 years, mean age 13 years, and the average length of follow up was 10.4 years. Fifty two per cent of the Down's syndrome patients were under 5 years of age, and $67 \%$ under 20 years, at the time of first recorded admission.

As expected, leukaemia was much more common in people with Down's syndrome than in the comparison cohort: the ratio in the former was 18.9 times higher than that in the latter (95\% confidence intervals (95\% CI): 10.4 to 31.5 ;

Table 1 Number of people with Down's syndrome and in the reference cohort admitted to hospital and matching ratio

\begin{tabular}{|c|c|c|c|c|}
\hline \multirow{2}{*}{$\begin{array}{l}\text { Age groups } \\
\text { (years) }\end{array}$} & \multicolumn{2}{|c|}{ Down's syndrome } & \multicolumn{2}{|c|}{ Reference cohort } \\
\hline & $\mathbf{n}$ & $\%$ & $\mathbf{n}$ & Matching ratio \\
\hline 0 & 573 & 39.4 & 27476 & 48 \\
\hline 1 & 72 & 5.0 & 17193 & 239 \\
\hline 2 & 44 & 3.0 & 11818 & 269 \\
\hline 3 & 43 & 3.0 & 12440 & 289 \\
\hline 4 & 22 & 1.5 & 14368 & 653 \\
\hline $5-9$ & 111 & 7.6 & 57054 & 514 \\
\hline $10-14$ & 72 & 5.0 & 40617 & 564 \\
\hline $15-19$ & 70 & 4.8 & 44941 & 642 \\
\hline $20-24$ & 59 & 4.1 & 48447 & 821 \\
\hline $25-29$ & 69 & 4.7 & 39788 & 577 \\
\hline $30-34$ & 59 & 4.1 & 31759 & 538 \\
\hline $35-39$ & 57 & 3.9 & 26442 & 464 \\
\hline $40-44$ & 60 & 4.1 & 23847 & 397 \\
\hline $45-49$ & 52 & 3.6 & 22919 & 441 \\
\hline $50-54$ & 55 & 3.8 & 22312 & 406 \\
\hline $55-59$ & 35 & 2.4 & 20785 & 594 \\
\hline Total & 1453 & 100.0 & 462206 & 318 \\
\hline
\end{tabular}

The table gives the number of people admitted to hospital with Down's syndrome in each age group stratum; the number of people in the reference cohort in each age group; and the number of people in the reference cohort per person with Down's syndrome in each age group stratum (matching ratio). table 2). A high rate ratio was found for both lymphoid and myeloid leukaemia. Of the 15 people with leukaemia, 11 were admitted aged less than 10 years. The rate ratio for leukaemia in people under 10 years of age with Down's syndrome, compared with people in the reference cohort with leukaemia aged less than 10 years was 31.0 (14.4 to 61.0). These figures exclude people with a first admission record for leukaemia and either Down's syndrome or reference condition (see Methods): there were five such people with leukaemia and Down's syndrome at the first admission. When the cases with simultaneous diagnoses were added to the analysis, the observed number of people with Down's and leukaemia was 20, the expected number was 0.9 , and rate ratio for leukaemia in the Down's cohort syndrome cohort compared with the reference cohort was 22.3 (95\% CI 13.5 to 35.0).

The risk ratio for all cancers combined, including leukaemia, was 2.7 ( 1.8 to 3.9). The risk ratio for cancers excluding leukaemia was 1.2 (0.6 to 2.2). Our results showed a significant increase in testicular cancer in people with Down's syndrome (table 2), albeit based on only three cases. No other cancer showed evidence of an increased risk associated with Down's syndrome, though numbers were small. The cancers that we analysed, but have not tabulated in this paper as there was only one case or none in the Down's cohort, were those of bladder, bone, brain, breast, cervix, kidney, liver, lung, nasopharynx, oesophagus, ovary, pancreas, prostate, rectum, salivary gland, skin, stomach, thyroid, upper respiratory tract, and uterus, and Hodgkin's lymphoma, multiple myeloma, and malignant melanoma.

People with Down's syndrome were significantly more likely than those in the reference cohort to have coeliac disease, acquired hypothyroidism, thyroiditis, thyrotoxicosis, chronic active hepatitis, and diabetes mellitus (table 3 ). Type 1 and type 2 diabetes mellitus are not distinguished as such in the dataset. In studying diabetes we therefore used an age cut off of less than 30 years, assuming that all (or almost all) such patients had type 1 diabetes. Diabetes mellitus under the age of 30 years was significantly more common in the Down's than in the reference cohort (rate ratio 2.8; 1.0 to 6.1). These figures excluded five people with a first admission record for both Down's syndrome and diabetes mellitus under age 30. When the simultaneous cases were included in the analysis, the rate ratio for diabetes under the age of 30 was 3.3 (95\% CI 1.8 to 5.4). Acquired hypothyroidism was significantly more common in people with Down's syndrome than the reference cohort (rate ratio: $9.4 ; 3.4$ to 20.5 ). These figures exclude people with a first admission record for both Down's syndrome and hypothyroidism: there were 15 such people. When the simultaneous cases were included in the analysis, the rate ratio for hypothyroidism in the Down's syndrome cohort was 29.5 times higher than in the comparison cohort (95\% CI 17.6 to 46.6). Asthma was significantly less common in people with Down's syndrome than in the reference cohort (rate ratio $0.4 ; 0.2$ to 0.6 ).

The immune related diseases that we studied, but that had one case or none in the Down's cohort, were Addison's disease, allergic dermatitis, ankylosing spondylitis, autoimmune haemolytic anaemia, dermatomyositis, Goodpasture's syndrome, idiopathic thrombocytopenia purpura, multiple sclerosis, myasthenia gravis, pemphigus, pernicious anaemia, polyarteritis nodosa, primary biliary cirrhosis, rheumatoid arthritis, scleroderma, Sjogren's syndrome, and systemic lupus erythematosus.

\section{DISCUSSION}

A strength of using the linked dataset is that some of the reporting and selection biases that may occur in interview based studies, based on clinical series at specialist centres, cannot occur in a population based, record based dataset. 
Table 2 Occurrence of cancer in people with Down's syndrome

\begin{tabular}{|c|c|c|c|c|c|}
\hline Cancer (ICD code)* & $\begin{array}{l}\text { Number in reference } \\
\text { cohort with each cancer }\end{array}$ & $\begin{array}{l}\text { Observed number in } \\
\text { Down's cohort }\end{array}$ & $\begin{array}{l}\text { Expected number in } \\
\text { Down's cohort }\end{array}$ & $\begin{array}{l}\text { Adjusted rate } \\
\text { ratio\# }\end{array}$ & $95 \% \mathrm{Cl}$ \\
\hline All cancers (140-208) & 9833 & 26 & 9.7 & 2.7 & 1.8 to 3.9 \\
\hline Leukaemia (204-208) & 348 & 15 & 0.8 & 18.9 & 10.4 to 31.5 \\
\hline Lymphoid leukaemia (204) & 181 & 11 & 0.5 & 22.2 & 10.9 to 40.6 \\
\hline Myeloid leukaemia (205) & 173 & 5 & 0.3 & 17.2 & 5.5 to 40.9 \\
\hline Lymphoma (200-202) & 520 & 2 & 0.8 & 2.7 & 0.3 to 9.6 \\
\hline Non-Hodgkin's lymphoma $(200,202)$ & 407 & 2 & 0.5 & 3.8 & 0.5 to 13.6 \\
\hline Colon (153) & 884 & 2 & 0.7 & 3.1 & 0.4 to 11.1 \\
\hline Testis (186) & 151 & 3 & 0.3 & 12.0 & 2.5 to 35.6 \\
\hline
\end{tabular}

*ICD 9 codes for each cancer (equivalent codes were used for cases coded in ICD Revisions 7, 8, and 10).

\#Adjusted for sex, age in 5 year bands (and in 1 year bands in those aged under 5 years), district of residence, and time period in single calendar years. The table gives the number of people with cancer in the reference cohort, the observed and expected number of people with cancer in the Down's syndrome cohort, the ratio of rates in the Down's syndrome cohort to that in the reference cohort, and $95 \% \mathrm{Cl}$ for the rate ratio.

Studies of associations between diseases are probably also subject to publication bias. ${ }^{12}{ }^{13}$ In this context, this is the bias that may occur if clinicians (and journal editors) regard a finding of significant association between two diseases more worthy of publication than a finding of no significant association between the same two diseases. This is particularly likely if there is no apparent reason to expect an association between the two diseases. Envisage one case series of disease $\mathrm{X}$ in which disease $\mathrm{Y}$ is observed, perhaps unexpectedly, to occur more commonly than in the general population; and another case series in which it does not occur more commonly. Typically, the first is more likely to be reported than the second: the finding in the second series would probably be considered unremarkable. A strength of our study is that we were able to study a range of cancers and immune related diseases within the same population of people with Down's syndrome. We report findings, both those that are positive and those that are not, from a single population across a range of conditions. Our results add strength to most of the findings summarised in Roizen and Patterson's literature review: they and we report associations between Down's syndrome and coeliac disease, thyroid disease, and diabetes. ${ }^{9}$ They suggested an association with rheumatoid arthritis which was not evident in the population studied by us. They did not comment on any association with asthma.

Our data also have some weaknesses. The cohorts are based on prevalent cases-the first recorded hospital admission or day case care for each person with each conditionrather than its being a cohort with follow up from birth. Data are not recorded on patients who move out of the area or who are treated in hospitals outside the region which is part of our reasoning for including a comparison cohort from the same database (see Methods). The data are based on hospital care and, whilst it is safe to assume that almost everyone with cancer will receive such care, many people with the immune related conditions, and with Down's syndrome itself, will not. For these reasons we cannot use our data to calculate absolute rates of disease occurrence. However, the calculation of relative risk of occurrence, comparing disease in those with Down's syndrome and the reference cohort, should be reliable with the following assumptions. We make the assumption that the extent of under-enumeration, as a result of using hospital data only, is similar in the Down's syndrome and reference cohort. We standardised the comparison between the Down's and reference cohorts for district and year of admission. We did this to try to make the two cohorts as comparable as possible in respect of clinical thresholds for admission (for example, for asthma or diabetes) which may vary by place and time. There is little or no information on other potential confounding factors or on clinical or cytogenetic characteristics of the patients. Finally, despite the size of the cohort, and the time span covered by it, the numbers of people with many of the diseases studied are still fairly small. This is particularly so for cancers that occur in the middle aged and elderly rather than the young.

\section{Cancer}

The level of risk of leukaemia found by us is very similar to the 20 -fold increase quoted in standard texts. ${ }^{12}$ It is noteworthy that the data in an administrative NHS statistics database can indeed be used to quantify such a risk reliably. Other studies of linked datasets have also identified the high

Table 3 Occurrence of autoimmune diseases in people with Down's syndrome

\begin{tabular}{|c|c|c|c|c|c|}
\hline $\begin{array}{l}\text { Immune related disease } \\
\text { (ICD code)* }\end{array}$ & $\begin{array}{l}\text { Number in reference cohort } \\
\text { with each immune related } \\
\text { disease }\end{array}$ & $\begin{array}{l}\text { Observed number } \\
\text { in Down's cohort }\end{array}$ & $\begin{array}{l}\text { Expected number } \\
\text { in Down's cohort }\end{array}$ & $\begin{array}{l}\text { Adjusted rate } \\
\text { ratiot }\end{array}$ & $95 \% \mathrm{Cl}$ \\
\hline Asthma (493) & 8846 & 20 & 51.7 & 0.4 & 0.2 to 0.6 \\
\hline Crohn's disease (555) & 662 & 3 & 1.2 & 2.6 & 0.5 to 7.7 \\
\hline Ulcerative colitis (556) & 652 & 3 & 0.9 & 3.3 & 0.7 to 9.6 \\
\hline Coeliac disease $(579.0)$ & 181 & 4 & 0.9 & 4.7 & 1.3 to 12.2 \\
\hline Diabetes mellitus under age $30(250)$ & 582 & 6 & 2.2 & 2.8 & 1.0 to 6.1 \\
\hline Thyrotoxicosis (242) & 387 & 5 & 0.7 & 7.4 & 2.4 to 17.4 \\
\hline Acquired hypothyroidism (244) & 413 & 6 & 0.6 & 9.4 & 3.4 to 20.5 \\
\hline Hashimoto's thyroiditis (245) & 40 & 2 & 0.04 & 44.1 & 5.2 to 170 \\
\hline Chronic active hepatitis (571.4) & 42 & 2 & 0.05 & 47.0 & 5.5 to 181 \\
\hline Psoriasis $(696.0,696.1,696.8,696.9)$ & 367 & 3 & 0.7 & 4.2 & 0.9 to 12.2 \\
\hline
\end{tabular}

*ICD 9 and equivalent codes in Revisions 7, 8, and 10

†Adjusted for sex, age in 5 year bands (and in 1 year bands in those aged under 5 years), district of residence, and time period in single calendar years. The table gives the number of people in the reference cohort, the observed and expected number of people with each disease in the Down's syndrome cohort, the ratio of rates in the Down's syndrome cohort to that in the reference cohort, and $95 \% \mathrm{Cl}$ for the rate ratio. ICD, International Classification of Diseases. 
risk of leukaemia. ${ }^{14-16}$ These findings support the validity of the study methods in assessing levels of risk for the other diseases studied.

It is sometimes considered that the risk of leukaemia is particularly high for acute myeloid leukaemia, but we found similarly high risks for both myeloid and lymphoid forms. Our data add some evidence to the suggestion that there is an increase in the risk of testicular cancer. The elevated risk of leukaemia can be considered in the broader context of how much the overall risk of cancer is increased: in our data, the overall risk is 2.9 times that of the general population in the age range and follow up periods studied.

\section{Immune related diseases}

As mentioned above, we corroborate the elevation of risk of coeliac disease and thyroid disease. ${ }^{9}$ The threefold increase in diabetes mellitus under the age of 30 years in people with Down's syndrome, is similar to that reported by others. ${ }^{8}$ Congenital hypothyroidism, as well as acquired hypothyroidism, is more common in Down's syndrome patients than the general population. We excluded the few people coded with congenital hypothyroidism from the analysis. We accept, however, that there may have been some diagnostic cross classification in our dataset which might have accounted for some of the strength of the observed association between hypothyroidism and Down's syndrome.

We found a significant deficit of asthma in people with Down's syndrome. Various explanations are possible and we cannot distinguish between them. It is possible that, although significant, this is in fact a chance finding or that it is attributable to uncontrolled factors in study design. If the deficit is real, one possibility is that people with Down's syndrome may be less predisposed than others to asthma. Another is that they may come into contact with asthma inducing allergens or pollutants less than other people. A third is that, if the hygiene hypothesis is correct, ${ }^{17}{ }^{18}$ they may have more infections than others early in life, with boosting of their immune systems and reduced antibody production against self antigens. Finally, people with Down's syndrome may have the same risk of asthma as other people but a lower rate of hospital admission. However, we think that, if anything, people with Down's syndrome would be more likely, rather than less likely, than those without to be admitted to hospital for a given level of symptoms. The findings on asthma need to be confirmed or refuted by other studies.

\section{ACKNOWLEDGEMENTS}

The database was built over many years by Leicester Gill and Glenys Bettley.

\section{Authors' affiliations}

M J Goldacre, C J Wotton, V Seagroatt, D Yeates, Unit of Health-Care Epidemiology, Department of Health, University of Oxford, Oxford, UK

The database was funded by the former Oxford Regional Health Authority. The Research and Development Directorate at the Department of Health funds the Unit of Health-Care Epidemiology to undertake research using the database.

Conflict of interest: none declared.

\section{REFERENCES}

1 Stiller CA. Malignancies. In: Pless IB, ed. The epidemiology of childhood disorders. Oxford: Oxford University Press, 1994:450-2.

2 Doll R, Peto R. Epidemiology of cancer. In: Weatherall D, Ledingham J, Warrell D, eds. Oxford textbook of medicine. 3rd ed. Vol 1. Oxford: Oxford University Press, 1996:214-5.

3 Dieckmann K-P, Rübe C, Henke R-P. Association of Down's syndrome and testicular cancer. J Urol 1997;157:1701-4.

4 Roberge D, Souhami L, Laplante M. Testicular seminoma and Down's syndrome. Can J Urol 2001;8(1):1203-6.

5 Book L, Hart A, Black J, et al. Prevalence and clinical characteristics of celiac disease in Downs syndrome in a U.S. study. Am J Med Gen 2001;98:70-4.

6 Bonamico M, Mariani P, Danesi HM, et al. Prevalence and clinical picture of celiac disease in Italian Down syndrome patients: a multicenter study. J Pediatr Gastroenterol Nutr 2001;33(2):139-43.

7 Tuysuz B, Beker DB. Thyroid dysfunction in children with Down's syndrome. Acta Paediatr 2001;90(12):1389-93.

8 Van Goor JC, Massa GG, Hirasing R. Increased incidence and prevalence of diabetes mellitus in Down's syndrome. Arch Dis Child 1997;77(2):186.

9 Roizen NJ, Patterson D. Down's syndrome. Lancet 2003;361:1281-9.

10 Goldacre M, Kurina L, Yeates D, et al. Use of large medical databases to study associations between diseases. QJM 2000;93:669-75.

11 Breslow NE, Day NE. Statistical methods in cancer research. Vol II. The design and analysis of cohort studies. IARC scientific publication No. 82. Lyon: International Agency for Research in Cancer, 1987:103-15.

12 Easterbrook PJ, Berlin JA, Gopalan R, et al. Publication bias in clinical research. Lancet 1991;337:867-72.

13 Last JM, ed. A dictionary of epidemiology. 4th ed. Oxford: Oxford University Press, 2001:145.

14 Hasle H, Clemmensen IH, Mikkelsen M. Risks of leukaemia and solid tumours in individuals with Down's syndrome. Lancet 2000;355:165-9.

15 Altmann AE, Halliday JL, Giles GG. Associations between congenital malformations and childhood cancer. A register-based case-control study. Br J Cancer 1998;78(9): 1244-9.

16 Boker LK, Blumstein T, Sadetzki S, et al. Incidence of leukaemia and other cancers in Down Syndrome subjects in Israel. Int J Cancer 2001;93:741-4.

17 Strachan DP. Hay fever, hygiene, and household size. BMJ 1999;299:1259-60.

18 Strachan DP. Family size, infection and atopy: the first decade of the "hygiene hypothesis". Thorax 2000;55(suppl 1):S2-10. 